\title{
Animal and vegetal poles of the mouse egg predict the polarity of the embryonic axis, yet are nonessential for development
}

\author{
Maria Anna Ciemerych ${ }^{1,2}$, Daniel Mesnard ${ }^{1}$ and Magdalena Zernicka-Goetz ${ }^{1, *}$ \\ ${ }^{1}$ Wellcome/CRC Institute and Department of Genetics, University of Cambridge, Tennis Court Road, Cambridge CB2 3HU, UK \\ 2Department of Embryology, Institute of Zoology, University of Warsaw, Krakowskie Przedmiescie 26/28, 00-927 Warsaw 64, \\ Poland \\ *Corresponding author (e-mail: mzg@mole.bio.cam.ac.uk)
}

Accepted 6 June; published on WWW 20 July 2000

\section{SUMMARY}

Recent studies suggest early (preimplantation) events might be important in the development of polarity in mammalian embryos. We report here lineage tracing experiments with green fluorescent protein showing that cells located either near to or opposite the polar body at the 8-cell stage of the mouse embryo retain their same relative positions in the blastocyst. Thus they come to lie on either end of an axis of symmetry of the blastocyst that has recently been shown to correlate with the anteriorposterior axis of the postimplantation embryo (see R. J. Weber, R. A. Pedersen, F. Wianny, M. J. Evans and M. Zernicka-Goetz (1999). Development 126, 5591-5598). The embryonic axes of the mouse can therefore be related to the position of the polar body at the 8-cell stage, and by implication, to the animal-vegetal axis of the zygote. However, we also show that chimeric embryos constructed from 2-cell stage blastomeres from which the animal or the vegetal poles have been removed can develop into normal blastocysts and become fertile adult mice. This is also true of chimeras composed of animal or vegetal pole cells derived through normal cleavage to the 8-cell stage. We discuss that although polarity of the postimplantation embryo can be traced back to the 8-cell stage and in turn to the organisation of the egg, it is not absolutely fixed by this time.

Key words: Mouse embryo, Polarity, Animal-vegetal axis, Egg, Blastocyst, Cell lineage, GFP

\section{INTRODUCTION}

In order to understand how spatial patterning arises in development it is critical to determine how the polarity of the embryo is first established. In many species, the organization of the early embryo bears a fixed relationship to the organization of the egg, and in some cases there is experimental evidence that specific regions of the egg are required for normal development. Eutherian mammals have been considered exceptional in this regard since normal animals are able to develop from preimplantation embryos manipulated in a way that would have been expected to perturb any polarity derived from the egg. Cleavage stage embryos can be aggregated to form chimeras, and individual blastomeres can be removed or displaced, without precluding their further development (for review see Papaioannou and Ebert, 1986; Pedersen, 1986; Gardner, 1998). Not surprisingly, the outcome of these manipulations has been taken as an indication that prepatterning of the mammalian egg is unlikely to provide cues for subsequent axis formation (for review see Gurdon, 1992).

Accordingly, the generally accepted viewpoint is that the anterior-posterior axis of the definitive embryo becomes specified only after implantation, shortly before gastrulation. This is when the anterior of the embryo can first be identified by molecular markers before the primitive streak forms at its future posterior aspect (reviewed by Beddington and Robertson, 1999; Davidson et al., 1999). However, it has recently become clear that visceral endoderm cells derived from inner cells near the polar body in the blastocyst preferentially colonise the embryonic region after implantation, moving to occupy the anterior of the embryo just before the onset of gastrulation (Thomas et al., 1998; Weber et al., 1999). Moreover, the polar body remains attached to the embryo during cleavage and occupies a stereotypic position aligned with the embryo's plane of bilateral symmetry at the blastocyst stage (Gardner, 1997). Together, these results suggest that the polar body might maintain a specific topographical connection to the animal pole of the egg during cleavage and imply that there might be a functional relationship between polar components of the egg and the polarity of the later embryo.

Although the regulative capacity of the mouse embryo is well established, it is only recently that there has been a direct test for the presence of determinants localised to specific polar regions of the egg. At the 1-cell stage, it has so far proved impossible to find a region that is essential for normal development (Zernicka-Goetz, 1998). It has been shown that the zygotes lacking either their animal or vegetal poles still can develop into live mice. However, these experiments would not have identified any polarised aspect of organization that might 
become localised at later stages of development (ZernickaGoetz, 1998; Gardner, 1999). Precedents for this type of mechanism are found in Xenopus and Caenorhabditis elegans where the final polarity of the egg is set up only after fertilisation. In Xenopus this is established by cortical rotation in response to sperm entry (Vincent and Gerhart, 1987). In $C$. elegans, sperm entry triggers cytoplasmic flux to establish an asymmetry on completion of the first cleavage and at the 2-cell stage this becomes evident when the plane of cleavage of one blastomere is altered by rotation of the mitotic spindle (for review see Bowerman, 1999). A role for cytoplasmic determinants in the development of the early mouse embryo has not been established (for review see Johnson et al, 1986). However, the early cleavage pattern in the mamalian embryo is regular, generally consisting of two meridional divisions followed by an equatorial one (Gulyas, 1975; Graham and Duessen, 1978; for review see Gardner, 1998). Thus, it could be argued that polarity of the embryo is established by regulating the plane of cleavage divisions that partition polar components or determinants of the egg. In this case, polarity might be specified as late as the 8-cell stage, the time at which blastomeres are derived from animal or vegetal egg cytoplasm as a consequence of the change of cleavage plane from meridional to equatorial.

To establish definitively whether there is a relationship between organisation of the zygote and any subsequent embryonic axes in the mouse, we wished to determine whether cell components and cells regularly remain in place during development. We addressed this by following lineages of cells from the 8-cell stage to the blastocyst. These lineage studies show that the polar body maintains a consistent relationship to the components of the embryo derived from the animal pole of the egg: the majority of blastomeres located either near to or opposite the polar body at the 8-cell stage retained this association in the blastocyst. Thus, in undisturbed development the orientation of the animal-vegetal axis of the egg does correlate with a major embryonic axis in the blastocyst and subsequently in the egg cylinder. To examine the hypothesis that any essential polarity might become fixed up to the 8-cell stage, we have followed the development of chimeras of several 2-cell blastomeres surgically deprived of their animal or vegetal poles components, or of 8-cell stage blastomeres naturally composed of animal pole-derived or vegetal polederived components. Both types of manipulated embryos are able to develop into blastocysts and then into fertile adult mice. Thus although polarity of the definitive embryo can be detected at the 8-cell stage and traced back to the organisation of the egg, it is capable of accomodating the removal of polar components even after fertilization, at both 2-cell and 8-cell stages.

\section{MATERIALS AND METHODS}

All embryos used to perform these experiments were collected from $\mathrm{F}_{1}$ (C57BL/6xCBA) females mated with males of the same genotype. 2-cell stage embryos were collected from the females ovulated spontaneously, on the morning of the second day after the presence of vaginal plug was confirmed. To control the timing of development from 4- to 8-cell stage, 4-cell embryos were collected from the females that were induced to ovulate by intraperitoneal injection of 7.5 IU of pregnant mares serum gondotrophin (PMSG, Intervet) followed 48 hours later by 7.5 IU of human chorionic gonadotrophin (hCG, Intervet) and then mated with males of the same genotype. Embryos were collected 54-56 hours after hCG injection. 8-cell stage embryos used to perform lineage studies were collected 70 hours after hCG injection.

Embryos were recovered after flushing of oviducts with Medium 2 (M2) containing $4 \mathrm{mg} / \mathrm{ml}$ of bovine serum albumin (M2 + BSA). The zonae pellucidae were then removed with the help of brief exposure to acid Tyrode's solution as previously described (Zernicka-Goetz et al., 1995). Zona-free embryos were always left for at least 30 minutes in KSOM supplemented with amino acids (KSOM + AA) and with 4 $\mathrm{mg} / \mathrm{ml}$ of bovine serum albumin (BSA; Speciality Media, Inc. Lavallette, NJ) before microsurgery.

\section{Lineage studies: microinjection, confocal microscopy and analysis}

After selection of late 8-cell stage embryos (approximately 70 hours following hCG injection) with intact polar bodies, a single blastomere was injected with a modified form of green fluorescent protein (GFP) mRNA using the method described previously (Zernicka-Goetz et al., 1997; Weber et al., 1999). We selected cells for injection that were located either near or opposite the polar body. As it is not possible to identify which of the three blastomeres that touch the polar body is actually attached to it (we could not detect transfer of GFP between the blastomere and the polar body) we followed lineages of one of these three cells. Following microinjection and then approximately 12 hours of incubation in $\mathrm{KSOM}$ at $37^{\circ} \mathrm{C}$, in an atmosphere of $5 \% \mathrm{CO}_{2}$, embryos showing green fluorescence were quickly selected using fluorescence microscopy. This step was used to eliminate embryos that were not labelled, together with those in which the clone was bigger than $1 / 8$ of the embryo. Then embryos were cultured in vitro until the blastocyst stage.

Embryos with a clearly visible polar body and showing normal development were analysed at the blastocyst stage. Each embryo, after being oriented, was observed by scanning confocal microscopy (Biorad). Images used for analysis corresponded to about 10 serial sections at 9 micron intervals of each embryo.

The distribution of the polar bodies was examined along three sectors of the embryonic-abembryonic axis, the mural, median and polar regions as defined by Gardner (1997). The position scored was where the polar body touches the blastocyst. The distribution of labelled cells was quantified with respect to three axes: the embryonicabembryonic axis; the animal-vegetal axis orthogonal to this in which the animal pole lies on the half of the embryo at which the polar body is present; and the left-right axis (Fig. 1A). Marked cells were counted at the early blastocyst stage when the spatial ratio of the blastocoele to the inner cell mass (ICM) is about 1/1. At this stage it is still possible to localise the polar body, whereas it becomes very flattened during blastocyst expansion and usually lyses shortly after.

\section{Removal of polar cytoplasm from the 2-cell stage embryo}

Two blastomeres of the 2-cell stage embryos were first gently separated from each other with a glass needle. Only blastomeres with attached polar bodies were then used for further manipulation. The removal of polar cytoplasm was completed in M2+BSA on an agarcoated Petri dish using a glass needle operated by hand as described previously (Tarkowski and Rossant, 1976; Zernicka-Goetz, 1998). The blastomeres were cut in groups of 10 and then transferred into the culture medium. The survival rate varied between experiments. In some experiments the majority of blastomeres survived, whereas in other cases as many as $30 \%$ of them lysed.

Blastomeres were cut in relation to the position of the polar body. The location of the polar body defines the animal pole of an embryo, and the diametrically opposite point on its surface is termed the vegetal pole (Balinsky, 1970). During the cutting operation the polar body remained attached to the blastomere and therefore provided a marker of the animal pole. In the first series of experiments the animal 
poles of the blastomeres were removed and then two or three blastomeres lacking these poles were aggregated with each other. In the second series of experiments the vegetal poles of blastomeres were cut away and then two or three blastomeres lacking vegetal poles were combined together. The percentage of cytoplasmic volume that was removed by this procedure was calculated from the diameter of the animal or vegetal fragments of the blastomere using IP Lab Spectrum image processing software (Scanalytics, USA). The proportion of cytoplasm removed when blastomeres were cut so as to discard animal or vegetal fragments was between 21 to $42 \%$ (average $33 \%$, 42 blastomeres) and 16 to $35 \%$ (average 27.5\%, 37 blastomeres) respectively. Reconstructed embryos were cultured in $\mathrm{KSOM}+\mathrm{AA}$ and BSA in atmosphere of $5 \% \mathrm{CO}_{2}$ at $37^{\circ} \mathrm{C}$, up to the morula/ blastocyst stage.

Late morulae or blastocysts derived from embryos lacking the animal or vegetal poles were transferred into the uteri of pseudopregnant mothers of the albino MF1 strain that had been mated 2.5 days earlier with vasectomised males. The experimental embryos were transferred together with 'carrier' blastocysts of MF1 strain of mice to control for the success of transfer. Such carrier embryos were contained within a zonae pellucidae, and had not been subjected to an in vitro culture.

\section{Animal or vegetal derived chimeras at the 8-cell stage}

Blastomeres of 4-cell embryos were separated from each other, using a glass needle, on an agar-coated Petri dish in M2+BSA. Only those blastomeres with attached polar bodies were used for further manipulation. They were placed separately in drops of KSOM + AA and BSA under paraffin oil and cultured in an atmosphere of $5 \% \mathrm{CO}_{2}$ at $37^{\circ} \mathrm{C}$ for the next $12-15$ hours until the third cleavage division had occurred. Blastomeres that cleaved and reached the 8-cell stage were scored, and only those pairs $(69 \%, 340 / 492)$ with the polar body attached to the end of one blastomere, well away from its contact point with the other blastomere, were used for further manipulations. Then, using a glass needle, they were separated from each other on an agarcoated Petri dish. These allowed us to obtain 1/8 blastomeres with attached polar bodies ('animal') and $1 / 8$ blastomeres without polar bodies ('vegetal'). Five to eight of either 'animal' or 'vegetal' blastomeres were aggregated with each other using phytohemagglutinin $(150 \mu \mathrm{g} / \mathrm{ml}$ in BSA-free M2 medium, Sigma) and then transferred into KSOM + AA and BSA. Reconstructed embryos were cultured up to late morula or blastocyst stages and then, along with MF1 carrier embryos, transferred into the uteri of pseudopregnant MF1 females as described above.

Embryos were photographed using a cooled CCD camera (Princeton Instruments, USA) and DIC optics on a Nikon inverted microscope.

\section{RESULTS}

\section{Polar body association of blastomeres at the 8-cell stage predicts a future embryonic axis in the blastocyst}

The mouse blastocyst has two morphologically recognisable axes. The first is the embryonic-abembryonic (EmAb) axis defined by the respective positions of the ICM and the blastocyst cavity. The outermost cells, the trophectoderm, can be divided into three regions: polar, median or mural depending upon where they lie along this axis (Fig. 1A). We define the second morphological axis as the 'long axis', which is perpendicular to the embryonic-abembryonic axis. This long axis is apparent because the external surface of the blastocyst has the shape of a slightly flattened ball - a prolate spheroid with the second polar body tending to be located at the end of its greater diameter (see legend to Fig. 1). It is in the plane of the equator that is positioned with respect to the embryonic and abembryonic poles. It is the longest straight line across the oval equatorial plane, and one end is usually closer to the polar body than the other. It will be shown that the orientation of the long axis approximates to the orientation of the animal-vegetal axis of the egg. This animal-vegetal axis lies on the plane of bilateral symmetry that passes through the polar body to divide both the trophectoderm and ICM into two symmetrical hemispheres.

We have assessed the position of the polar body along the embryonic-abembryonic axis of the blastocyst following the same criteria as Gardner (1997) (see Materials and Methods). We found that in most cases the polar body came to lie in the median region of the blastocyst: in 43\% (19/44) of embryos the polar body was present within the median region itself and in $10 \%(4 / 44)$ of embryos the polar body was found on the border between the median region and either the polar or mural region. This is a similar proportion to that found by Gardner $(54 \%)$ in his study (Gardner, 1997). In 27\% (12/44) of blastocysts, the polar body was located towards the polar region (overlying the polar trophectoderm) and in 20\% (9/44) of blastocysts the polar body was found in the mural region. Thus, in 53\% of the blastocysts the polar body was found on one third of the embryo surface (the median surface) and was no more than $15^{\circ}$ on either side of a long axis end.

The polar bodies from the meiotic divisions define the animal pole of the egg (Balinsky, 1970). The animal-vegetal (AV) axis of the egg passes from the area occupied by the polar body to a point directly opposite on the egg's surface. Previous work had shown that the second polar body was attached to the membrane of a blastomere during development to the blastocyst (Gardner, 1997). We wished to determine further, by cell lineage analysis, whether the polar body retained its position in relation to the components of the blastomeres throughout development from the 8-cell stage to the blastocyst. To this end we injected single blastomeres near the polar body in a total of 328 -cell stage embryos and single blastomeres on the opposite side of the embryo in 29 8-cell embryos with synthetic mRNA for GFP to trace their fate in vitro until the blastocyst stage. In 44 injected embryos (25 with blastomeres labelled near and 19 with blastomeres labelled opposite the polar body) we could clearly identify the position of the polar body in the blastocyst thus allowing us to analyse the distribution of the final clones of GFP-expressing cells.

The striking feature of our results is that the labelled cells remained tightly clustered together in clones that showed a strong relationship between their location and the position of the polar body (Fig. 1B). When the embryo had been injected in a cell opposite the polar body, the labelled clone was found opposite the polar body in the blastocyst. Conversely, in embryos injected in a cell near the polar body, the final clone was also found near the polar body. Our primary interest was to determine the distribution of clones between animal and vegetal hemispheres of the blastocyst in the two groups. As there is some variation in the final position of the polar body upon the plane of bilateral symmetry, there are two ways of defining the animal and vegetal halves of the blastocyst. In the first, the position of the clone can be scored with respect to the animal hemisphere that has the polar body at the center of its curved surface. In blastocysts arising from 8-cell embryos 
A

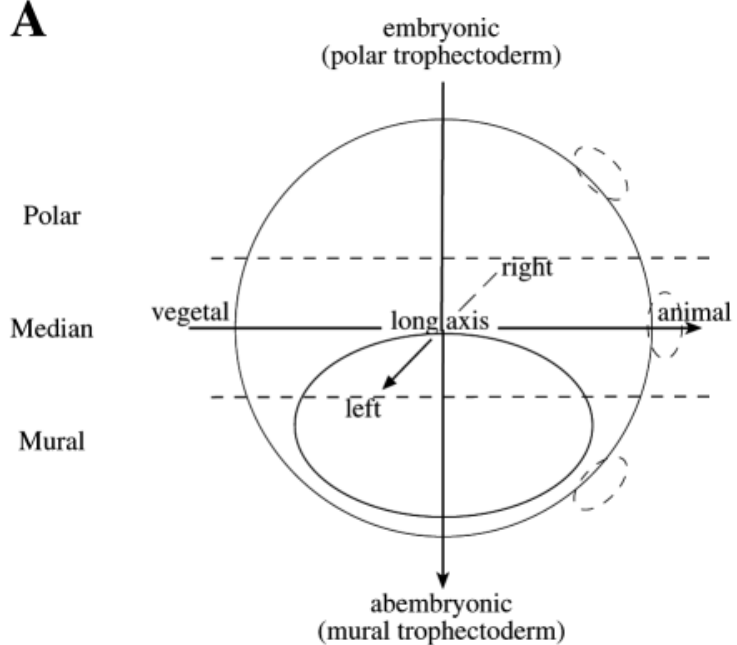

B

Polar PB
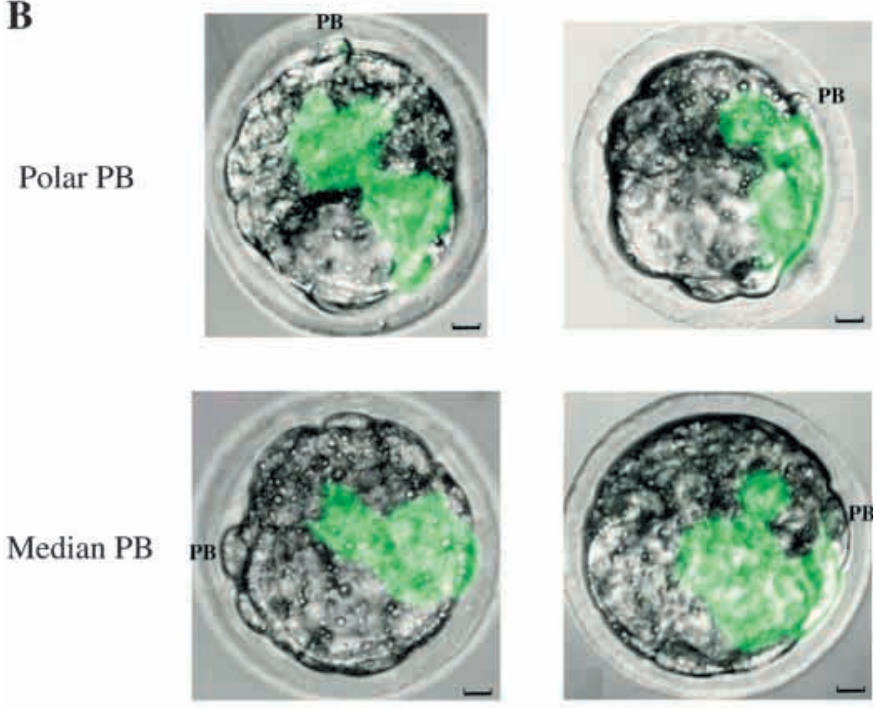

Mural PB

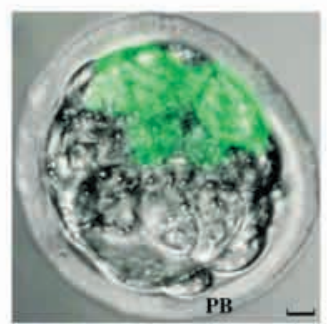

Injection opposite the $\mathrm{PB}$

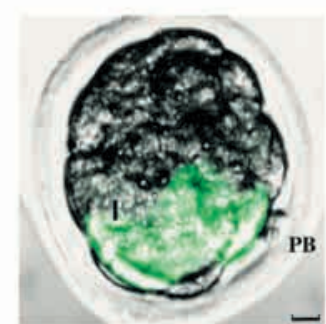

Injection near the PB injected near the polar body, $68 \%$ of cells in all of the labelled clones (25 embryos analysed) were located in the animal hemisphere, as defined by the above criterion. Conversely, in blastocysts derived from 8-cell embryos injected opposite the polar body only $18 \%$ of cells from the labelled clones (19 embryos analysed) extended into the animal half.

The distribution of cells between the two hemispheres defined in the above manner may vary, depending upon the relative position of the polar body and the blastocyst cavity. Therefore, we also analysed our data with respect to another plane at right angles to the plane of bilateral symmetry that cuts the blastocyst into hemispheres with approximately equal
Fig. 1. Clonal localisation in relation to the polar body position. (A) The diagram shows the conventions used for the three axes of the blastocyst, and the distribution of the polar body (PB) along three sectors of the embryonic-abembryonic axis. At the stage of analysis our embryos contained an average of 56 cells $( \pm 9.4)$ of which $34 \%$ $(19.2 \pm 5.2), 43 \%(24.2 \pm 4.5)$ and $23 \%(12.6 \pm 4.8)$ were distributed between the polar, median and mural regions respectively, with 8-10 cells in the ICM. We determined the long (animal-vegetal) axis of the blastocyst to be $94.4 \mu \mathrm{m}( \pm 5.5 \mu \mathrm{m})$ and the short (left-right) axis, $88.1 \mu \mathrm{m}( \pm 4.3 \mu \mathrm{m})$. (B) The micrographs show projected series of 10 optical sections that illustrate the relative positions of the labelled clones with regard to the polar body, following injection near or opposite the PB. The position of the PB is marked on each micrograph. The size of the clones (6-8 cells labelled) is consistent with the injected cells having undergone 3 rounds of cell division. At the stage examined there were no differences in the size of the clones in the two groups of embryos. On average $67 \%$ of clones contributed to both ICM and trophectoderm, and 33\% only to trophectoderm. Scale bars, $10 \mu \mathrm{m}$.

numbers of trophoblast and ICM cells. To score the distribution of clones with respect to the blastocyst structure, the embryo was divided into two hemispheres with the ends of the long axis in the center of each of their curved surfaces. We found that irrespective of the position of the polar body in relation to the embryonic-abembryonic axis, the descendants of cells located near the polar body at the 8-cell stage showed a pronounced tendency $(71 \%)$ to remain on the animal hemisphere of the blastocyst axis (Fig. 2A). Conversely, cells located opposite the polar body at the 8-cell stage gave rise to progeny of which only $29 \%$ were found in the animal hemisphere of the blastocyst as defined above (Fig. 2B).

It is apparent from the above data that cells within clones derived from the single injected cell remain in place to the blastocyst stage. This is supported by a number of other observations. If we examine the proportion of cells occupying the polar region of the embryonic-abembryonic axis descended from a 1/8 blastomere near the polar body, we find it reflects the final position of the polar body along this axis. So when the polar body is polar, $66 \%$ of cells are polar; when it is median, $55 \%$ of cells are polar; and when it is mural, $33 \%$ are polar (Fig. 2C). We see the converse relationship in the proportion of polar cells descended from a 1/8 blastomere opposite the polar body $(45 \%, 73 \%$, and $92 \%$, of polar cells when the polar body lies in the respective polar, median and mural regions; Fig. 2D).

Thus the principal finding of our lineage tracing using GFP is that cytoplasmic components of the $1 / 8$ cells associated with the polar body retain this association until the blastocyst stage. The long axis of the blastocyst is therefore related to the animal-vegetal axis of the zygote. It also shows that in unperturbed development the polar body and its neighbouring cells come to occupy a polarized position on the developing embryonic axis. These findings advocate the hypothesis that polar components of the early embryo are distributed together with the polar body into the blastocyst in a stereotypic manner.

\section{Animal and vegetal associated clones can extend along the embryonic-abembryonic axis of the early blastocyst}

We wished to examine the distribution of clones along the two other principal axes of the blastocyst: the 'left-right' axis 

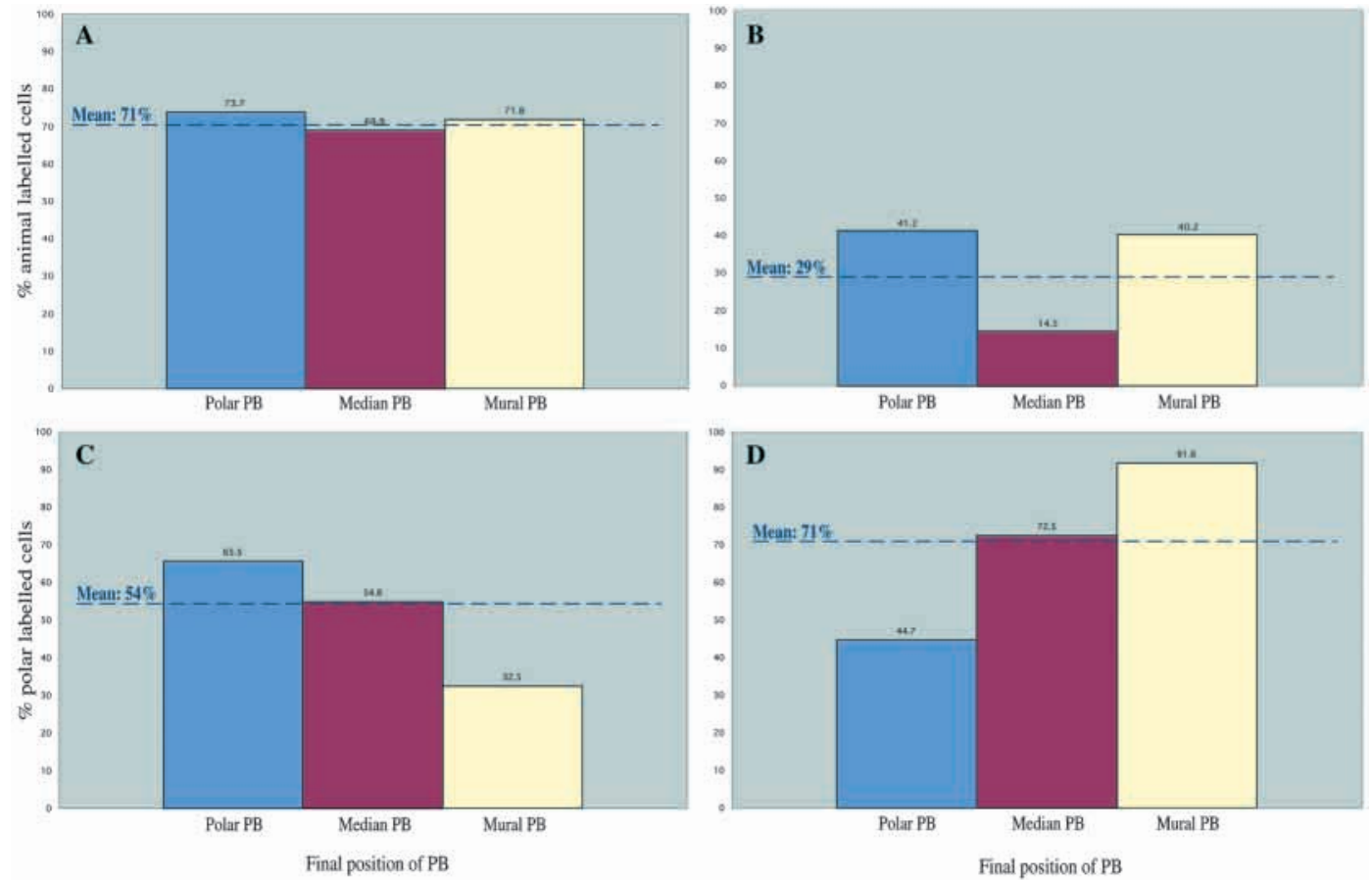

Fig. 2. Contribution of labelled cells from either end of the animal-vegetal axis at the 8-cell stage to the animal half (A,B) or polar half (C,D) of the blastocyst. Histograms showing the proportion at the blastocyst stage of labelled animal cells in relation to the PB position, following injection near (A) or opposite (B) the PB at the 8-cell stage. The horizontal axis is divided according to the final position of the PB along the EmAb axis: polar (blue), median (red), and mural (yellow). From 44 labelled embryos we could analyse 39; 5 embryos were excluded from this analysis as the PB was located on the EmAb axis thus the relative orientation of the long axis could not be defined. The total number of embryos analysed after injection near to the PB was 21 and after injection opposite the PB was 18. (C,D) Histograms showing the proportion of labelled cells in the polar region of the blastocyst in relation to the PB position at this stage, following injection near (C) or opposite (D) the PB at the 8-cell stage. The dashed blue line shows the global mean of labelled cells.

about the plane of bilateral symmetry, and the embryonicabembryonic axis (Fig. 1A). We could see differences between the distribution of clones coming from 8-cell embryos injected either near to or opposite the polar body to the left and right hemispheres. In embryos injected near the polar body, $49 \%$ of cells came to lie on the left half and $51 \%$ on the right half of the blastocyst. In embryos injected opposite the polar body, $59 \%$ of cells become located to the left and $41 \%$ to the right halves of the blastocyst. Thus, this axis of symmetry appears to bear no relationship to the AV axis.

Interestingly, the proportion of labelled cells that distribute between polar, median and mural regions is not significantly different for embryos injected in cells near the polar body $(27 \%, 42 \%, 31 \%$ respectively) or opposite the polar body (30\%, 43\%, 27\% respectively). Moreover, in embryos from both groups $60 \%$ of the labelled clones encompass at least two of these three regions of the embryonic-abembryonic axis, and $40 \%$ of clones extend over all three regions. In neither case do we see differences between the two groups along the EmAb axis of the early blastocyst indicating firstly that at this stage of development there is no detectable relative 'movement' of 'animal' or 'vegetal'-derived clones with respect to this axis and secondly that the two groups of clones can be found along the embryonic-abembryonic axis of the early blastocyst. As an alternative way of looking at this question, we examined the extent to which clones of trophectoderm would cross the mural-polar borders, the equator, in the total data set. We found that in the early blastocyst only $34 \%$ of clones lie exclusively in one or other region whereas the majority of clones $(66 \%)$ extend over the two, supporting the previous conclusion.

\section{Removal of polar cytoplasm from the 2-cell stage embryo does not perturb development}

The major finding of the above lineage studies affirms that the animal-vegetal axis that is represented by the position of the polar body at these early cleavage stages is reflected in the later axes. This might appear inconsistent with our earlier work showing that removal of the animal or vegetal cytoplasm from the mouse egg shortly before cytokinesis did not prevent their further development (Zernicka-Goetz, 1998). Gardner (1999) has argued, however, that those experiments do not disprove the critical role of egg polarity, since any cytoplasmic rearrangements initiated by fertilization might not be completed until just before the first cleavage of the zygote. But if this were the case, cutting away the animal or vegetal pole of an early 2-cell stage embryo could impair its developmental potential.

To determine whether the polar cytoplasm of the 2-cell stage mouse embryo is required for development, we removed either the animal or the vegetal pole of the $1 / 2$ blastomere by cutting eggs perpendicular to the animal-vegetal axis. It appeared technically difficult to cut both blastomeres of the 2-cell stage embryo precisely in the same plane when they remained attached to each other. Therefore, we first separated the blastomeres from each other, and then cut only the cell to which the polar body was attached, because the AV axis in this 
A
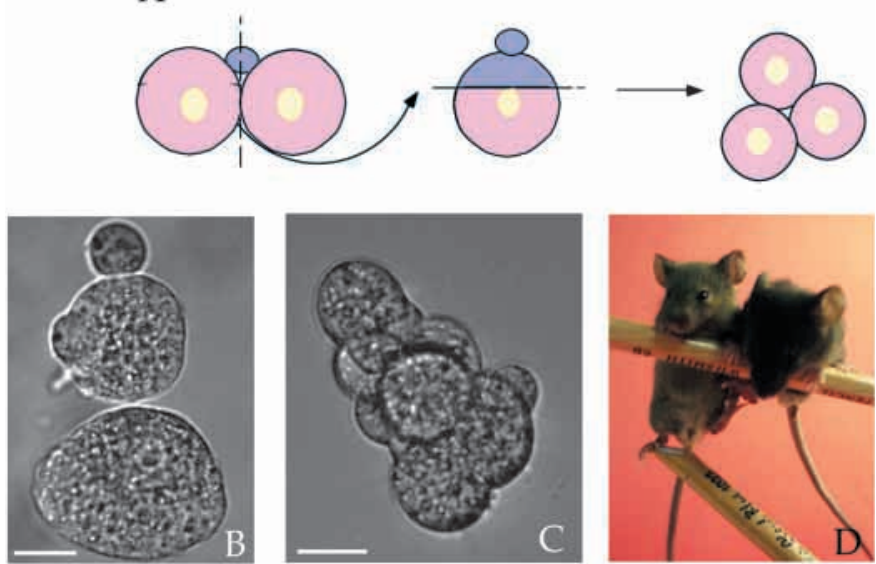

Fig. 3. Development of an embryo lacking the animal pole.

(A) Scheme to illustrate the method of separation of blastomeres, bisection and aggregation. Colour is used to indicate the area of the blastomere that was cut away, not the location of determinants.

(B) Transmitted light image of a representative 1/2 blastomere after cutting to remove the animal pole. The animal fragment with its attached PB was discarded, and the vegetal fragment was used to produce chimeras ( 2 or 3 vegetal fragments combined together). (C,D) Subsequent development of a 'vegetal' chimera to the 8-cell stage (C) and newborn mice (D). Scale bars, $25 \mu \mathrm{m}$.

case could be precisely identified (Figs $3 \mathrm{~A}$ and $4 \mathrm{~A}$ ). This microsurgery was carried out in such a way that one of the resulting fragments of the cell contained the nucleus (Figs 3B and $4 \mathrm{~B}$ ). This bisection removed, on average, $33 \%$ or $28 \%$ of the total blastomere volume from the animal or vegetal part of the blastomere respectively (see Materials and Methods). Altogether from 12 independent experiments we obtained 110 blastomeres lacking their animal poles and 78 lacking their vegetal poles. As we knew from our previous experiments that reducing the volume of the blastomere cytoplasm would reduce the number of cells within the embryo and so would compromise its developmental potential (Zernicka-Goetz, 1998), we aggregated two or three of the surgically manipulated blastomeres to make chimeras containing only cells missing cytoplasm from one or other pole. After aggregation, embryos were cultured in vitro for 3 days. We found that $32 / 41$ (78\%) of chimeric embryos missing animal pole, and 15/21 (71\%) of chimeric embryos missing vegetal pole, developed into morulae (Fig. 3C) and subsequently to blastocysts (Fig. 4C).

To test definitively the developmental potential of such 'animal' and 'vegetal' embryos, we determined their capacity to complete embryogenesis to term. Blastocysts derived from both types of embryos were therefore transferred to the uteri of foster mothers, together with genetically distinct carrier embryos that had neither been manipulated nor cultured in vitro. Of embryos that were transferred to the uteri of recipient mothers that became pregnant and gave birth, 9 out of $15(60 \%)$ lacking the animal pole, and 2 out of 4 from those lacking the vegetal pole, developed normally to term (Figs 3D, 4D). To ensure that there were also no determinants at the poles that were necessary for the development of the germ line, we verified that the mice derived from embryos lacking specific poles were fertile. Moreover, dissection of these animals
A
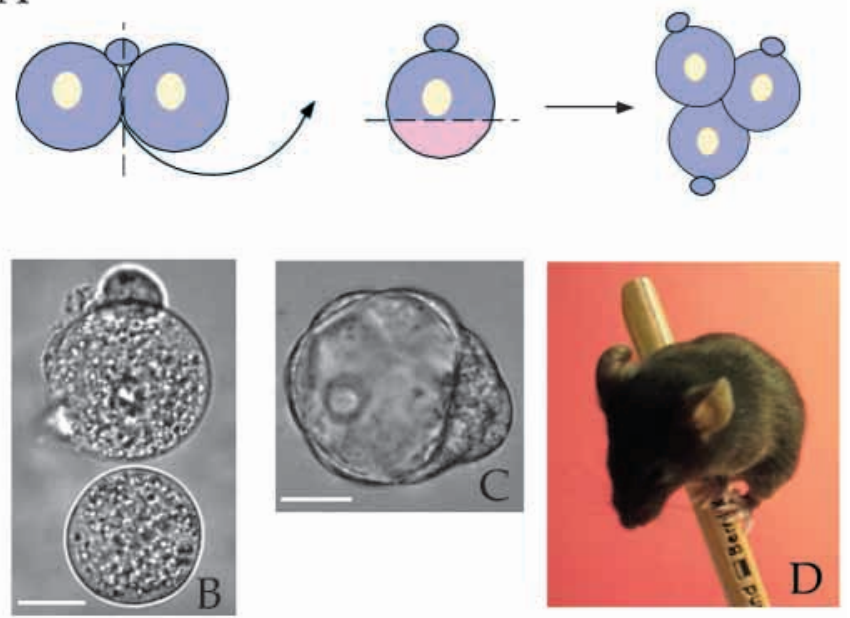

Fig. 4. Development of an embryo lacking the vegetal pole. (A) Scheme to illustrate the method of separation of blastomeres, bisection and aggregation. (B) Transmitted light image of a representative $1 / 2$ blastomere after cutting to remove the vegetal pole. The vegetal pole was discarded, and the animal fragment was used to produce chimeras. (C,D) Subsequent development of an 'animal' chimera to the blastocyst (C) and newborn mice (D). Scale bars, $25 \mu \mathrm{m}$.

revealed that their internal organs were positioned in correct locations as in control mice.

\section{Animal and vegetal derived blastomeres at the 8-cell stage have full developmental potential}

If we assume that during cleavage the structure of the zygote was compartmentalised into different cells without any major rearrangement of its components, then the pattern of cleavage described for mouse development suggests that the 8-cell embryo is the earliest stage at which individual blastomeres will consist of only the animal or vegetal parts of the zygote (for review see Gardner, 1998). Normal development has never been observed from individual blastomeres from 8-cell mouse embryos, but this could be attributed to the fact that the individual blastomeres isolated at that stage were unable to produce a sufficient number of cells to form the ICM at the blastocyst (Tarkowski and Wroblewska, 1967). Alternatively, it could mean that both animal and vegetal derived blastomeres are required for development.

Therefore to test whether there is a developmentally significant consequence of animal-vegetal polarity that is acquired by the 8-cell stage, we reconstructed embryos composed of cells derived from only animal or only vegetal material. Because blastomeres change their orientation during cleavage it is impossible to distinguish precisely which blastomeres at the 8-cell stage are animal or vegetal, with the exception of the one that is attached to the polar body. So to be able to generate not only animal but also vegetal chimeras at the 8-cell stage, we collected blastomeres attached to the polar body from 4-cell stage embryos and cultured them in vitro for the 12-15 hours to the next cleavage division (Fig. 5A, scheme). In 69\% (340/492) of such isolated blastomeres cleavage was equatorial (Fig. 5B) to give animal $1 / 8$ blastomeres, which were separated from their vegetal sisters. 


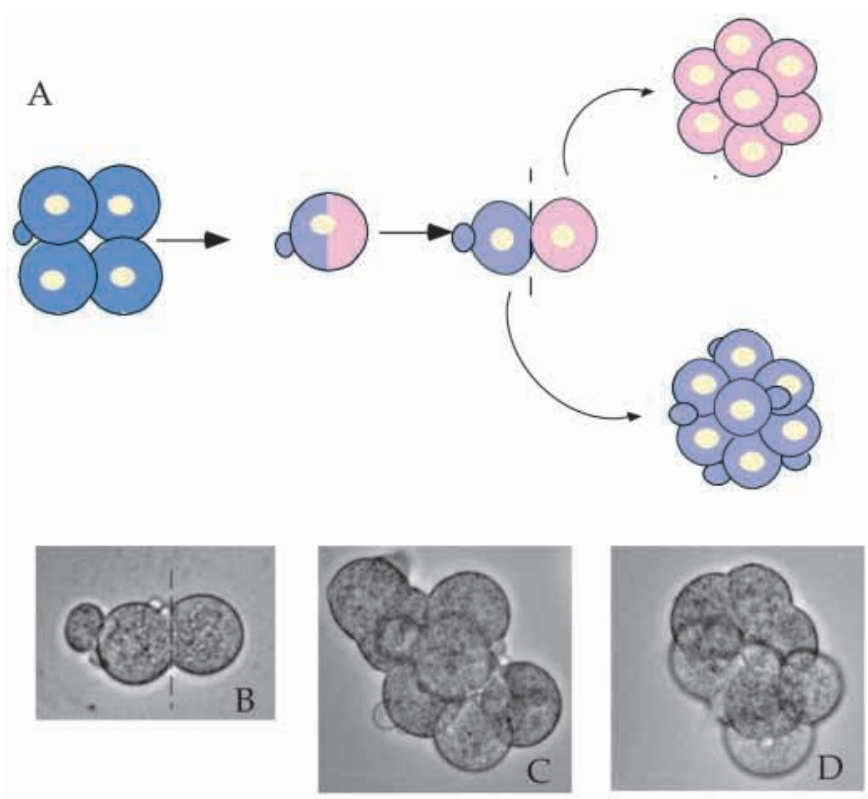

Fig. 5. (A) Scheme to illustrate the method of production of 'animal' or 'vegetal' chimeras at the 8-cell stage. Colour is used to indicate the halves of the cell that were separated from each other, not the location of determinants. (B) Transmitted light image of a representative blastomere after the third cleavage division. Polar body is attached to the animal $1 / 8$ blastomere. (C,D) Subsequent development of the 'animal' (C) and 'vegetal' chimeras into morulae. Magnification, 225 $\times$.

Altogether from 15 independent experiments we collected 340 animal $1 / 8$ blastomeres and 302 vegetal $1 / 8$ blastomeres. These were combined (5-8 blastomeres together) separately to generate animal-derived and vegetal-derived chimeras (Fig. 5C,D). Chimeric embryos were cultured in vitro for another 30 hours and we found that embryos of both types could develop into morulae/blastocysts.

To test developmental potential of these 'animal' and 'vegetal' embryos they were transferred into the uteri of pseudopregnant mothers and respectively $31 \%$ (14 out of 45) and $21 \%$ ( 6 out of 29 ) developed to term. In these experiments $65 \%$ (41 out of 63) of unmanipulated carrier MF1 embryos were born. The proportion of 'animal' and 'vegetal' embryos that developed to term in our studies is comparable to the proportion of embryos developing to term in other reported experiments in which chimeric embryos were produced (for example Brown et al., 1990; Dvorak et al., 1995). Mice that developed from either 'animal' or 'vegetal' chimeras also proved to be fertile.

\section{DISCUSSION}

It has been relatively easy to see a relationship between the localised components of eggs and the structure of the resulting embryos in several animal species, largely because the eggs have or soon develop conspicuous regionalisation of cytoplasmic components. This has not been possible in mice. Nevertheless, we are able to infer that such a relationship exists from the data we now present. Assuming there is no major rearrangement of cytoplasmic components, the previously observed pattern of cleavage of the mouse embryo would typically divide animal pole cytoplasm from vegetal pole at the 8-cell stage. Our tracing of the fate of animal or vegetal blastomeres from this stage using GFP as a lineage marker establishes that in normal development this animalvegetal axis is conveyed to the long axis of the blastocyst that lies on the plane of bilateral symmetry, and this, in turn, is related to the major body axis of the mouse. We show that cell components and cells regularly remain in place during development from the 8-cell stage to the blastocyst. Consequently clones of cells derived from blastomeres near to the polar body at the 8-cell stage come to be located close to the polar body of the blastocyst, some 3 cell divisions later. These studies show that the polar body and its neighbouring cells come to occupy a polarized position on the developing embryonic axis. In approximately half of the embryos, the polar body comes to lie within the median region on one end of the long axis of the blastocyst. However, even when the polar body is situated a lesser or greater distance from the embryonic pole, clones of 1/8 blastomeres initially positioned near the polar body are found to preferentially occupy the 'animal hemisphere' of the blastocyst as defined in relation to its long axis. The final link to the body axis of the embryo proper is given by previous lineage studies that showed this long axis of the blastocyst to be further transformed, after implantation, first into the proximodistal axis of the egg cylinder and then into the anterior-posterior axis of the embryo (Weber et al., 1999).

Despite the existence of such a correlation between the zygote and embryonic axes, our experiments using chimeric embryos comprising either animal pole- or vegetal polederived cells show that essential polarity has not been rigidly fixed by the 8-cell stage. These data extend previous findings (Zernicka-Goetz, 1998) showing that eggs from which the poles had been surgically removed had full developmental potential. However, since those experiments removed the poles of the eggs shortly after fertilization, they could not exclude the possibility that polarity was functionally established only subsequently. This could occur either late in the first cell cycle by cytoskeletal or cytoplasmic rearrangement triggered by fertilization, as seen in both Xenopus and C. elegans, or as further elaborated in $C$. elegans by the defined pattern of early cleavages.

The possibility that the characteristic cleavage pattern of the mouse embryo is critical for setting up the future body plan appears to be diminished by our experiments on 8-cell stage embryos. The reported pattern of cleavage should create blastomeres derived from either animal or vegetal egg components at the 8-cell stage. Our finding that such isolated animal or vegetal blastomeres from 8-cell stage embryos have full developmental potential following aggregation into 'animal' and 'vegetal' chimeras shows that neither the animal nor the vegetal poles of the 8-cell embryo contain components that are essential for the development of polarity in the later embryo. Although one cannot be sure in these experiments of the extent to which blastomeres might rotate during the first cleavage divisions, thus potentially distorting the proportions of animal or vegetal cytoplasm in the blastomere, we can be certain of the identity of the cell attached to the polar body. This of course would assume that there was no movement of the cytoplasm or membrane with respect to each other. Our 
lineage tracing from the 8-cell stage onwards indicates for the first time that cytoplasmic components remain associated with the membrane.

Thus, there is an apparent contradiction between this conclusion on the one hand and the evidence on the other hand that there is a continuity between organization of the egg, the blastocyst, and subsequently the polarity of the postimplantation embryo as outlined above. If we conclude that the relationship between the long axis of the blastocyst and the animal-vegetal axis of the egg is coincidental it is difficult to explain this continuity of fate. So we are driven to the alternative conclusion that while the mouse embryo may use the patterning of the zygote in its normal development, effective regulative mechanisms are able to re-establish polarity even after such drastic perturbations as the ones described here. Such a mechanism may take time to reestablish, and consistent with this we have observed a developmental delay of up to 1 day in such manipulated embryos until a morphologically recognizable primitive streak can been seen in the post-implantation embryo.

Nevertheless, the mechanisms that establish polarity in the mouse embryo may be related to the original organization of the zygote. There could be, for example, a morphogenetic gradient in the egg that relies on the relative and not the absolute concentration of a morphogen. In this case, even after one pole of the egg is removed, the gradient could persist and still be able to direct proper development. It is also possible that either remaining pole of the egg is able to define polarity because it contains information that identifies it as one end of an axis of symmetry, or that the sub-polar region can re-specify polar character when the original pole is removed. Our studies thus emphasize not only the importance of identifying the mechanism and timing of the establishment of polarity in normal development, but also of identifying when polarity is irreversibly determined in the embryo, i.e., the time at which regulative mechanisms are no longer effective should the embryo be perturbed.

In conclusion, the studies presented here indicate that although the embryonic axes are already evident within the organization of the zygote, they are not irreversibly established at this stage. In fact neither the animal nor the vegetal pole of the cleaving embryo is absolutely required for the morphogenesis of the blastocyst, or for the axial development of the definitive embryo. Therefore, it remains important to understand the underlying significance of egg organisation in normal, undisturbed development to reveal the nature of the extensive regulative properties of the mouse embryo, and to learn the extent of similarities in axis-determining mechanisms among vertebrates.

We are grateful to our colleagues for support and helpful discussions throughout the course of this work. Lister Institute of Preventive Medicine Fellowship, Wellcome Trust Project Grant and Royal Society Equipment Grant to M. Z.-G. supported this work. We also thank the anonymous reviewers of this paper for their very valuable suggestions.

\section{REFERENCES}

Balinsky, B. I. (1970). An Introduction to Embryology. Philadelphia: W. B. Saunders Company.

Beddington, R. S. and Robertson, E. J. (1999). Axis development and early asymmetry in mammals. Cell 96, 195-209.

Bowerman, B. (1999). The maternal control of polarity and patterning during embyogenesis in the nematode Caenorhabitis elegans. In Cell Lineage and Fate Determination (ed. S. A. Moody), pp. 97-117. San Diego: Academic Press.

Brown, N. A, McCarthy and A. and Wolpert, L. (1990). The development of handed assymetry in aggregation chimeras of situs inversus mutant and wild-type mouse embryo. Development 110, 949-954.

Davidson, B. P., Camus, A. and Tam, P. L. (1999). Cell fate and lineage specification in the gastrulating embryo. In Cell Lineage and Fate Determination (ed. S. A. Moody), pp. 491-504. San Diego: Academic Press.

Dvorak P., Yoshiki, A., Dvorakova, D. Flechon, J. E. and Kusakabe, M. (1995). Cell mixing during the early development of mouse aggregation chimera. Int. J. Dev. Biol. 39, 645-652.

Gardner, R. L. (1997). The early blastocyst is bilaterally symmetrical and its axis of symmetry is aligned with the animal-vegetal axis of the zygote in the mouse. Development 124, 289-301.

Gardner, R. L. (1998). Axial relationships between egg and embryo in the mouse. Curr. Top. Dev. Biol. 39, 35-71.

Gardner, R. L. (1999). Scrambled or bisected mouse eggs and the basis of patterning in mammals. BioEssays 21, 271-274.

Graham, C. F. and Deussen, Z. A. (1978). Features of cell lineage in preimplantation mouse development. J. Embryol. Exp. Morphol. 48, 53-72.

Gulyas, B. J. (1975). A reexamination of cleavage patterns in eutherian mammalian eggs: rotation of blastomere pairs during second cleavage in the rabbit. J. Exp. Zool. 19, 235-248.

Gurdon, J. B. (1992). The generation of diversity and pattern in animal development. Cell 68, 185-199.

Johnson, M. H. (1986). A role for cytoplasmic determinants in the development of the mouse early embryo? J. Embryol. Exp. Morphol. 97, 97211.

Papaioannou V. E and Ebert K. M. (1986). Comparative aspects of embryo manipulation in mammals. In: Experimental Aproaches to Mammalian Embryonic Development, (J. Rossant and R. A. Pedersen, eds.)., Cambridge University Press, New York, pp. 67-90.

Pedersen, R. A. (1986). Potency, lineage, and allocation in the preimplantation mouse embryo. In: Experimental Aproaches to Mammalian Embryonic Development (J. Rossant and R. A. Pedersen, eds.), pp. 3-33. Cambridge University Press, New York,

Tarkowski, A. K and Wroblewska, J. (1967). Development of blastomeres of mouse eggs isolated at the 4- and 8-cell stage. J. Embryol. Exp. Morphol. 18, $155-180$.

Tarkowski, A. K. and Rossant, J. (1976). Haploid mouse blastocysts developed from bisected zygotes. Nature 259, 663-665.

Thomas, P., Brown, A. and Beddington, R. S. P. (1998). Hex: a homeobox gene revealing peri-implantation asymmetry in the mouse embryo and an early transient marker of endothelial cell precursors. Development 125, 85 94.

Vincent, J. P. and Gerhart, J. C. (1987). Subcortical rotation in Xenopus eggs: an early step in embryonic axis specification. Dev. Biol. 123, 526-39.

Weber, R. J., Pedersen, R. A., Wianny, F., Evans, M. J. and ZernickaGoetz, M. (1999). Polarity of the mouse conceptus is established before implantation. Development 126, 5591-5598.

Zernicka-Goetz, M. (1998). Fertile offspring derived from mammalian eggs lacking either animal or vegetal poles. Development 125, 4803-4808.

Zernicka-Goetz, M., Ciemerych, M. A., Kubiak, J. Z., Tarkowski, A. K. and Maro, B. (1995). Cytostatic factor inactivation is induced by a calciumdependent mechanism present until the second cell cycle in fertilized but not in parthenogenetically activated mouse eggs. J. Cell Sci. 108, 469-474.

Zernicka-Goetz, M., Pines, J., McLean-Hunter, S., Dixon, J. P. C., Siemering, K., Haseloff, J. and Evans, M. J. (1997). Following cell fate in the living mouse embryo. Development 124, 1133-1137. 